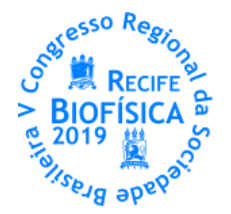

\title{
A INTERAÇÃO ANALITO-NANOPORO GERA UM PROCESSO ESTOCÁSTICO MARKOVIANO OU COM MEMÓRIA?
}

\author{
Gesilda F. Neves ${ }^{1 *}$, Dijanah C. Machado², Carlos M. M. Carneiro², Luiz H. A. Consoni², Cláudio G. Rodrigues², Romildo \\ A. Nogueira ${ }^{1}$
}

\begin{abstract}
${ }^{1}$ Departamento de Morfologia e Fisiologia Animal, Universidade Federal Rural de Pernambuco, ${ }^{2}$ Departamento de Biofísica e Radiobiologia, Universidade Federal de Pernambuco, Recife, PE, Brasil

*gesildaflorenco@gmail.com
\end{abstract}

\section{INTRODUÇÃO}

0 biossensoriamento estocástico é uma abordagem que se baseia na observação de eventos de interação entre moléculas individuais de analitos e um único biorreceptor, que pode ser, por exemplo, um nanoporo biológico (AGUIAR et al., 2015). A denominação estocástico para esse biossensoriamento é atribuída, principalmente, em relação à série temporal de flutuações discretas registradas, devido à detecção, por um único nanoporo, de cada molécula do analito, portanto, as moléculas são detectadas uma a uma, ou seja, de maneira unitária ou individual (AGUIAR et al., 2015; MACHADO et al., 2016). 0 poro formado pela alfatoxina provavelmente é a nanoestrutura biológica mais utilizada para fins de sensoriamento estocástico. A alfatoxina é uma exotoxina secretada pela bactéria Staphylococcus aureus, na forma de monômeros polipeptídicos contendo 293 aminoácidos, que se oligomerizam em membranas lipídicas formando um nanoporo heptamérico (KRASILNIKOV et al., 1981; MENESTRINA et al., 1986). O maior interesse no nanoporo formado por esta toxina ocorreu devido a possibilidade do desenvolvimento de um método para sequenciamento de material genético, uma vez que ela permite a translocação de DNA de fita simples (KASIANOWICZ et al.,1996; HOWORKA et al., 2001; AGAH et al., 2016). Isto permitiu a fabricação de plataformas de sequenciamento de terceira geração (www.nanopore.com). Devido a sua estabilidade estrutural e elevada condutância, o nanoporo da alfatoxina também tem sido empregado na detecção e análise do processo de translocação de polímeros neutros, como por exemplo, o polietilenoglicol (RODRIGUES et al., 2008; RODRIGUES et al., 2011; MACHADO, et al., 2016). Estudos da influência da força iônica no processo de translocação do polietilenoglicol através deste nanoporo gerou um método não destrutivo para determinação do tamanho de moléculas poliméricas em meio aquoso (ROBERTSON et al., 2007). O sensor formado pela alfatoxina consiste na inserção de um único nanoporo em uma membrana artificial banhada por uma solução eletrolítica. 0 mecanismo de sensoriamento do nanoporo baseia-se na aplicação de um potencial elétrico transmembrana, que induzirá uma corrente iônica máxima através do poro; as moléculas do analito ao entrarem no nanoporo, bloqueiam parte dessa corrente iônica gerando uma série temporal de eventos discretizados (BAYLEY \& CREMER, 2001; AGUIAR et al., 2015). Neste contexto, cada evento reflete a interação da molécula unitária com o nanoporo e a análise da série temporal, ou seja, determinação dos tempos caraterísticos de ocupação ou de ausência, bem como a amplitude dos eventos discretizados (bloqueios), permitem a identificação da molécula permeante, pois, representam uma assinatura digital do analito (LASZLO et al.,
2016). Os modelos atuais preconizam que a série temporal dos eventos de bloqueio da corrente iônica resultantes da interação analito-nanoporo sejam regidas por leis que descrevem processos estocásticos (COLQUHOUN \& HAWKES, 1995). Técnicas para estudo de correlação de longo alcance foram aplicadas ao registro das séries de abertura e fechamento de canais iônicos, através da análise de Hurst há alguns anos (CAMPOS DE OLIVEIRA et al., 2006), possibilitando estudar a presença ou não da memória em canais. Siwy e colaboradores (SIWY; AUSLOOS; IVANOVA, 2002) estudaram a série temporal de abertura e fechamento de canais BK para verificar o comportamento da correlação e neste caso, foram maiores que 0,5 e fortes correlações foram identificadas nos estados fechados. Lan et al. (2008) mostram a possibilidade de ser estudada a corrente de canais de $\mathrm{K}^{+}$dependente de voltagem em neurônios ganglionares de ratos através de DFA. Em canais BK também foram estudados memória e gates (WAWRZKIEWICZ et al., 2012). A correlação, isto é, a memória de longa duração no decorrer do tempo, é definida quando um evento passado tem efeito sobre eventos futuros (NOGUEIRA, R. A.; VARANDA, W. A.; LIEBOVITCH, L. S.,1995; CAMPOS DE OLIVEIRA et al., 2006; LAN et al., 2008;WAWRZKIEWICZ et al., 2012). O método amplamente utilizado para identificar correlações de longo alcance, curto alcance e aleatoriedade em séries temporais é a Análise de Flutuação Destendenciada - DFA (PENG et al. 1994), considerado eficaz para medir a dependência de longo alcance para séries temporais não-estacionárias (CHIARUCCI; RUZZENENTI; LOFFREDO, 2014). Esta tem sido aplicada em diversos campos, tais como a dinâmica cardíaca, biologia molecular, economia, clima, ciência dos materiais, eletrofisiologia, entre outros (HU et al., 2001; CABALLERO et al., 2002; CHEN et al., 2002; LAN et al., 2008). Neste contexto, investigamos se a série temporal de corrente iônica resultante da interação do polietilenoglicol monodisperso de peso molecular 1294 (PEG 1294) com o nanoporo de alfatoxina é um processo estocástico markoviano ou com memória.

\section{Experimental}

\section{MATERIAIS E MÉTODOS}

Todas as bicamadas lipídicas planas livres de solvente foram confeccionadas com diftanoilfosfatidilcolina, $2 \%(\mathrm{p} / \mathrm{v})$ em hexano, conforme metodologia padrão para construção de membranas lipídicas artificiais (MONTAL \& MUELLER, 1972). Todos os reagentes foram de grau analítico e usados sem qualquer processamento adicional. O lipídeo sintético foi adquirido da Avanti Polar Lipids (Alabaster, AL). A alfatoxina foi comprada da Calbiochem (Alemanha). O polietilenoglicol (PEG1294) monodisperso (Polypure, Oslo, Norway) foi usado como um analito representativo de 
polímeros neutros por causa de sua ampla aplicação em diversas áreas. As concentrações de PEG 1294 utilizadas nos experimentos foram de 400 e $4000 \mu \mathrm{M}$. A solução banhante da membrana era composta por $\mathrm{KCl} 4 \mathrm{M}$, Tris $5 \mathrm{mM}$, pH 7,5. Todos os experimentos foram realizados a $25 \pm 1{ }^{\circ} \mathrm{C}$, em condições de fixação de voltagem (potencial de $20 \mathrm{a} 100 \mathrm{mV}$, incremento de $20 \mathrm{mV}$ ) e as membranas apresentaram capacitância de aproximadamente $40 \mathrm{pF}$. Todos os registros de corrente iônica transmembrana foram processados por filtro Bessel em $15 \mathrm{kHz}$ e armazenadas diretamente na memória de um microcomputador.

\section{Teórico}

O sinal de corrente iônica foi tratado no software QUB (www.qubbuffalo.edu) e os tempos de permanência nos estados bloqueado (TP) e não-bloqueado (TA) foram obtidos separadamente e analisados através do algoritmo de DFA. A extração dos tempos de permanência nos estados TA e TP possibilita determinar o perfil comportamental da série em cada um desses estados (Figura 1).

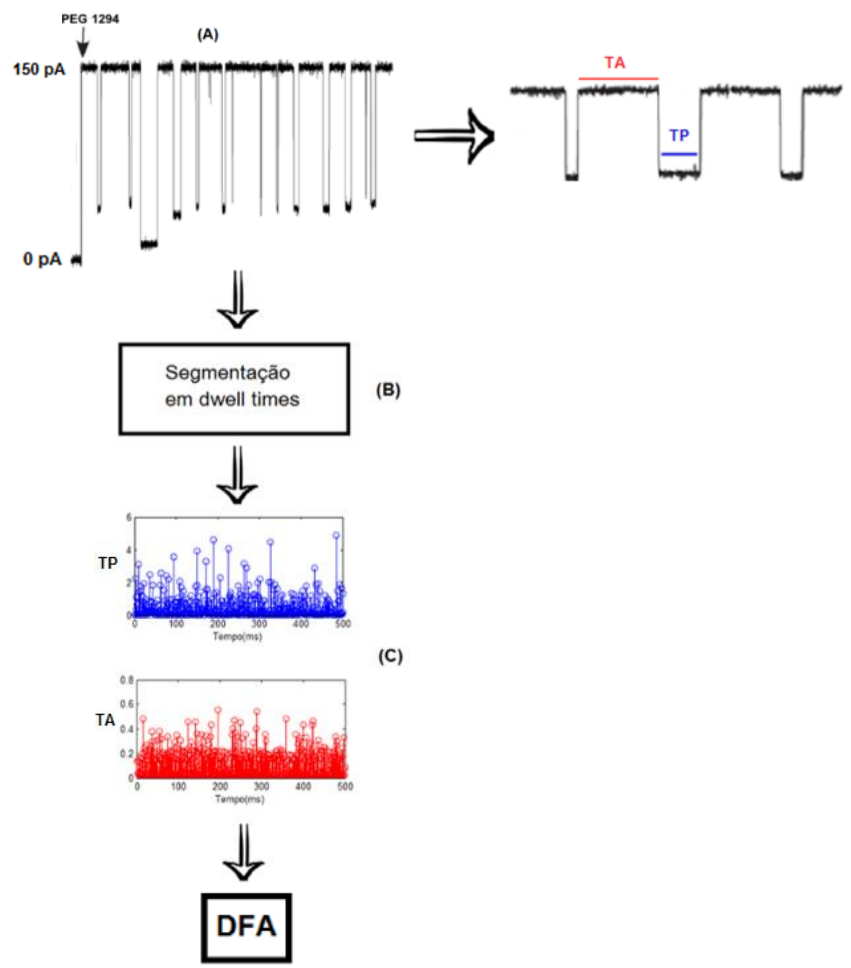

Figura 1. (A) registro do sinal de corrente iônica e os estados TA (tempo de ausência do analito no nanoporo) e TP (tempo de presença do analito no nanoporo); (B) segmentação do sinal de corrente em tempos de permanência num determinado estado(dwell times) através do software QUB; (C) dwell times separados para análise da correlação através do DFA.

Análise de Flutuação Destendenciada. 0 método DFA baseia-se em análise de flutuações dos dados após a retirada das tendências da série temporal integrada. 0 procedimento é o seguinte:

(a) seja y(i) com $\mathrm{i}=1,2, \ldots, \mathrm{N}$, a série temporal original, a série integrada é obtida usando a expressão:

$$
y(k)=\sum_{i=1}^{k}(y(i)-M)
$$

onde, $M$ é a média dos valores de $y(i)$ com $i=1,2, \ldots, N$ e $k$ é um número inteiro. A integração transforma a série original em um processo auto-similar;

(b) Em seguida, a série $\mathrm{y}(\mathrm{k})$ é dividida em intervalos de tamanho $\mathrm{n}$. A ordenada do segmento que representa a tendência no intervalo de tamanho $\mathrm{n}$ é denotada $\mathrm{y}_{\mathrm{n}}(\mathrm{k})$. Essa tendência é retirada subtraindo-se o valor do ajuste $\mathrm{y}_{\mathrm{n}}(\mathrm{k})$ do valor correspondente da série integrada $\mathrm{y}(\mathrm{k})$. A função de flutuação $\mathrm{F}(\mathrm{n})$ que caracteriza o tamanho das flutuações para o tamanho $\mathrm{n}$ dos intervalos usados, é dada pela expressão:

$$
F(n)=\sqrt{\frac{1}{N}} \sum_{K=1}^{N}\left[y(k)-y_{n}(k)\right]^{2}
$$

$\mathrm{O}$ procedimento é repetido para vários tamanhos do intervalo $\mathrm{n}$ para poder determinar a relação entre a função de flutuação $F(n)$ e o tamanho do intervalo $n$. Como $F(n)$ versus $n$ segue uma lei de potência com expoente de auto-similaridade $a$, este expoente a pode ser calculado usando o coeficiente angular da reta obtida por regressão linear do gráfico log $F(n)$ versus $\log (n)$ (PENG et al.,1995).

O parâmetro de autossimilaridade a tem a interpretação seguinte (PENG et al., 1995):

a) 0 valor $0<a<0,5$ indica as correlações de longo alcance antipersistentes;

b) Para ruído branco (série não correlacionada) $\alpha=0,5$;

c) 0 valor $0,5<a<1$ indica as correlações de longo alcance persistentes;

d) 0 caso $a=1$ representa ruído do tipo $1 / f$;

e) Para $a>1$ correlações ainda existem, mas não se comportam pela lei de potência. 0 caso especial $a=1,5$ indica ruído Browniano que representa a integração do ruído branco.

\section{RESULTADOS E DISCUSSÃO}

Na figura 2 apresentamos o resultado da análise do DFA nos tempos de presença (TP) e ausência (TA) oriundas da série temporal da corrente iônica do nanoporo da alfatoxina na presença de PEG 1294 nas concentrações de 400 e $4000 \mu \mathrm{M}$, sob aplicação do potencial de $+40 \mathrm{mV}$. Nossos resultados corroboram com diversos estudos, demonstrando que a taxa de transição para formação do complexo PEG-nanoporo é diretamente proporcional à concentração do polímero, com uma inclinação de valor unitário (MOVILEANU et al., 2003; RODRIGUES et al., 2008; ZHAO et al., 2008; NESTOROVICH et al., 2010). Isto sugere que a partição do PEG no nanoporo pode ser descrita por uma reação de primeira ordem entre o poro e o polímero e que apenas uma molécula de polímero é responsável por um único evento de bloqueio. A representação gráfica do DFA para as séries temporais estudadas neste trabalho consiste em um processo estocástico tanto nos estados de TA quanto TP. Apesar da existência de diversos estudos com nanoporo (RINCON-RESTREPO et al., 2011; KIM \& DUNBAR, 2016), ainda não está completamente elucidado quais os mecanismos moleculares envolvidos no processo de transporte de moléculas no interior do nanoporo de alfatoxina, os quais influenciam na interação analito-nanoporo e consequentemente nos tempos cinéticos do nanoporo-sensor (Zhao 2008). A análise da série temporal dos eventos de bloqueio de corrente iônica induzidos pela interação analito-nanoporo por meio de métodos não-lineares, como o DFA, pode ajudar a responder alguns questionamentos. A aplicação do DFA para investigar a cinética de canais iônicos foi mostrada inicialmente por Lan e cols. (2008), em seu trabalho apresentaram a possibilidade de estudar a corrente de canais de $\mathrm{K}^{+}$dependente de voltagem em neurônios glangionares de ratos através de DFA e modelo Markoviano. 

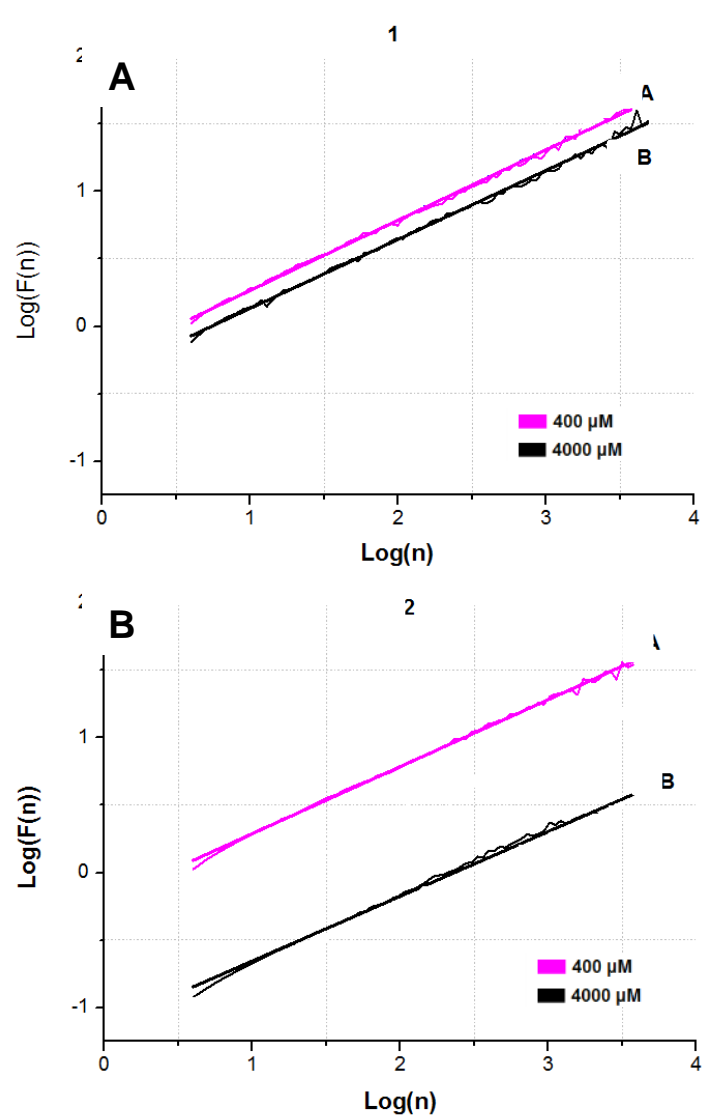

Figura 2. Resultado do alfa do DFA na condição de aplicação de voltagem $+40 \mathrm{mV}$. (A) Valores de TA foram: 0,521 e 0,511 para as concentrações de 400 e $4000 \mu \mathrm{M}$, respectivamente. (B) Valores de TP foram: 0,496 e 0,480 para as concentrações de 400 e $4000 \mu \mathrm{M}$, respectivamente.

A tabela 1 mostra os valores dos alfas do DFA para os TP e TA do nanoporo na presença de diferentes concentrações de PEG 1294 nas concentrações de 400 e $4000 \mu \mathrm{M}$ e sob aplicação de potencial que variou de $+20 \mathrm{a}+100 \mathrm{mV}$ (incremento de $20 \mathrm{mV}$ ). Pode-se observar que os valores dos alfas do DFA, tanto para TP como TA, em sua dinâmica de bloqueios e desbloqueios foram em todas as condições experimentais investigadas aproximadamente iguais a 0,5 .

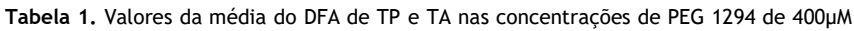
e $4000 \mu \mathrm{M}$, sob aplicação de potencial de $+20 \mathrm{a}+100 \mathrm{mV}$.

\begin{tabular}{ccccc}
\hline & \multicolumn{2}{c}{$400 \mu \mathrm{M}$} & \multicolumn{2}{c}{$4000 \mu \mathrm{M}$} \\
\cline { 2 - 4 } & \multicolumn{2}{c}{ TP/TA } & \multicolumn{2}{c}{ TP/TA } \\
\hline $20 \mathrm{mV}$ & 0,497 & 0,521 & 0,510 & 0,512 \\
\hline $40 \mathrm{mV}$ & 0,505 & 0,511 & 0,510 & 0,513 \\
\hline $60 \mathrm{mV}$ & 0,507 & 0,505 & 0,511 & 0,513 \\
\hline $80 \mathrm{mV}$ & 0,532 & 0,513 & 0,493 & 0,504 \\
\hline $100 \mathrm{mV}$ & 0,492 & 0,506 & 0,500 & 0,529 \\
\hline
\end{tabular}

Neste estudo, através do uso do DFA foi possível inferir que o comportamento do nanoporo da alfatoxina e sua interação com o PEG 1294 consiste em um processo aleatório em que TA e TP concentram-se em torno de um alfa de 0,5. Para descrever um comportamento estocástico sem memória e mimetizar os comportamentos de bloqueio e desbloqueio de canais iônicos utiliza-se a modelagem Markoviana. Através desta ferramenta, foi possível descrever numa modelagem de dois estados, os TA e TP do nanoporo nas condições estudadas neste trabalho (LAN et al., 2005; LAN et al., 2008). A modelagem Markoviana consiste de um pequeno conjunto de estados, das conexões entre eles e das taxas cinéticas, que são as probabilidades do poro mudar de um estado para outro. Os resultados mostraram que um modelo Markoviano de dois estados cinéticos (1 TA e 1 TP) descreve bem a cinética do nanoporo e condizem com os experimentais confirmando a não existência de memória no processo, portanto bloqueio estocástico (Figura 3).

A

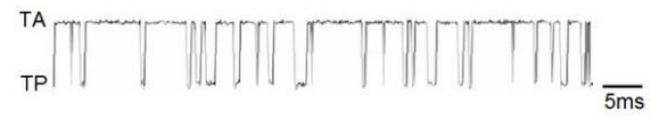

B

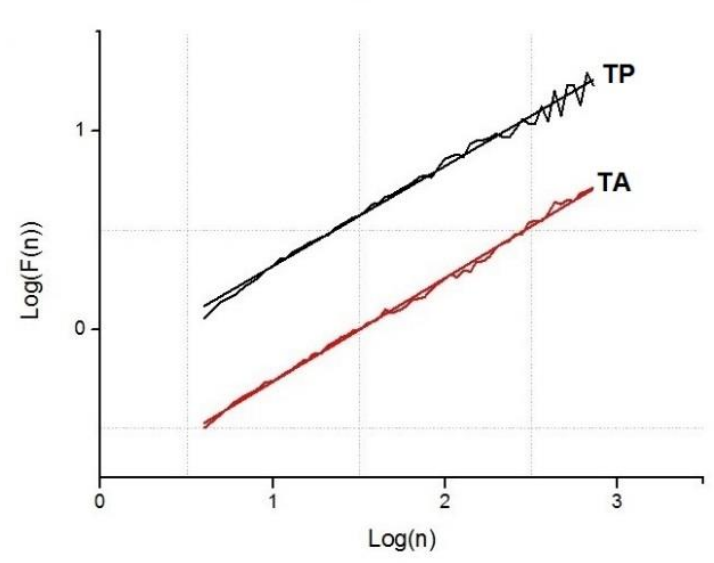

Figura 3. (A) Modelagem Markoviana de 2 estados para a simulação do registro experimental (PEG 1294 a $400 \mu \mathrm{M}$, voltagem aplicada $+40 \mathrm{mV}$ ). (B) alfa de TP $(0,50548)$ e TA $(0,52241)$.

\section{CONCLUSÕES}

Verificamos que a translocação do PEG 1294 no tempo de chegada e no tempo de permanência dentro do nanoporo segue a dinâmica de um processo estocástico Markoviano. Mais estudos serão realizados para ajudar a descrever em detalhes o comportamento deste processo.

\section{REFERÊNCIAS}

AGAH, S. et al. DNA sequencing by nanopores: Advances and challenges. Journal of Physics D: Applied Physics, v. 49, n. 41, p. 413001, 2016.

AGUIAR, J. P., JÚNIOR, J. J. S., MACHADO, D. C. \& MELO, M. C. A. Biossensoriamento estocástico via nanoporo proteico individual no desenvolvimento de ferramentas analíticas. Quim. Nova, n.38, p.817-827, 2015.

BAYLEY, H. \& CREMER, P. S. Stochastic sensors inspired by biology. Nature, n.413, p.226-230, 2001.

CABALLERO, R., JEWSON, S. \& BRIX, A. Long memory in surface air temperature: Detection, modeling, and application to weather derivative valuation. Clim. Res., n. 21, p. 127-140,2002.

CHEN, Z., IVANOV, P. C., HU, K. \& STANLEY, H. E. Effect of nonstationarities on detrended fluctuation analysis. Phys. Rev. E Stat. Nonlinear, Soft Matter Phys.,n. 65,p. 1-15, 2002.

CHIARUCCI, R.; RUZZENENTI, F.; LOFFREDO, M. I. Detecting spatial homogeneity in the World Trade Web with Detrended Fluctuation Analysis. Physica A: Statistical Mechanics and its Applications, v. 401, p. 1-7, 2014.

COLQUHOUN, D.; HAWKES, A. G. The principles of the stochastic interpretation of ion-channel mechanisms. In: Single-channel recording. Springer, Boston, MA, 1995. p. 397-482. 
HOWORKA, S., CHELEY, S. \& BAYLEY, H. Sequence-specific detection of individual DNA strands using engineered nanopores. Nat. Biotechnol., n. 19, p.636-639, 2001.

KASIANOWICZ, J. J., BRANDIN, E., BRANTON, D. \& DEAMER, D. W. Characterization of individual polynucleotide molecules using a membrane channel. Proc. Natl. Acad. Sci. U.S.A., n. 93, p. 13770$3,1996$.

KIM, J. \& DUNBAR, W. B. High-precision low-power DNA readout interface chip for multichannel nanopore applications. Sensors Actuators, B Chem., n. 234, p.273-277, 2016.

Krasilnikov, O. V.; Ternovskii, V. I; Tashmukhamedov, B. A; Biofizika 1981,26,271

LAN, T. H., XI, H. \& LIN, J. R. Correlation character of ionic current fluctuations: Analysis of ion current through a voltagedependent potassium single channel. Biophys. Chem., n.117, p. 191-197,2005.

LAN, T.-H., GAO, Z.-Y., ABDALLA, A. N., CHENG, B. \& WANG, S. Detrended fluctuation analysis as a statistical method to study ion single channel signal. Cell Biol. Int., n. 32, p.247-252,2008.

LASZLO, A. H., DERRINGTON, I. M. \& GUNDLACH, J. H. MspA nanopore as a single-molecule tool: From sequencing to SPRNT. Methods, n. 105, p. 75-89,2016.

MACHADO, D. C. et al. Effects of alkali and ammonium ions in the detection of poly(ethyleneglycol) by alpha-hemolysin nanopore sensor. RSC Adv., n. 6, p. 56647-56655,2016.

MENESTRINA, G. Ionic channels formed by Staphylococcus aureus alpha-toxin: Voltage-dependent inhibition by divalent and trivalent cations. The Journal of membrane biology, v. 90, n. 2, p. 177190, 1986.

MONTAL, M.; MUELLER, P. Formation of bimolecular membranes from lipid monolayers and a study of their electrical properties. Proc. Natl. Acad. Sci. U.S.A., v. 69, n. 12, p. 3561-3566, 1972.

MOVILEANU, L.; CHELEY, S.; BAYLEY, H. Partitioning of individual flexible polymers into a nanoscopic protein pore. Biophysical Journal, v. 85, n. 2, p. 897-910, 2003.

NESTOROVICH, E. M. et al. Blockage of anthrax PA63 pore by a multicharged high-affinity toxin inhibitor. Biophysical Journal, v. 99, n. 1, p. 134-143, 2010.

NOGUEIRA, R. A.; VARANDA, W. A.; LIEBOVITCH, L. S. Hurst analysis in the study of ion channel kinetics. Brazilian Journal of Medical and Biological Research , v. 28, n.4, p. 491-496, 1995.

PENG, C.-K. et al. Fractal mechanisms and heart rate dynamics: long-range correlations and their breakdown with disease. Journal of Electrocardiology, v. 28, p. 59-65, 1995.

RINCON-RESTREPO, M. et al. Controlled translocation of individual DNA molecules through protein nanopores with engineered molecular brakes. Nano letters, v. 11, n. 2, p. 746-750, 2011.

ROBERTSON, J. W.F. et al. Single-molecule mass spectrometry in solution using a solitary nanopore. Proc. Natl. Acad. Sci. U.S.A., v. 104, n. 20, p. 8207-8211, 2007.

RODRIGUES, C. G. et al. Mechanism of $\mathrm{KCl}$ enhancement in detection of nonionic polymers by nanopore sensors. Biophysical Journal, v. 95, n. 11, p. 5186-5192, 2008.

RODRIGUES, C. G. et al. Mechanism of $\mathrm{KCl}$ enhancement in detection of nonionic polymers by nanopore sensors. Biophysical Journal, v. 95, n. 11, p. 5186-5192, 2008.

RODRIGUES, C. G. et al. Hofmeister effect in confined spaces: halogen ions and single molecule detection. Biophysical Journal, v. 100, n. 12, p. 2929-2935, 2011.

SIWY, Z.; AUSLOOS, M.; IVANOVA, K. Correlation studies of open and closed state fluctuations in an ion channel: Analysis of ion current through a large-conductance locust potassium channel. Physical review. E, Statistical, nonlinear, and soft matter physics, v. 65, n. 3, p. 6, 2002.
WAWRZKIEWICZ, A. et al. On the simple random-walk models of ion-channel gate dynamics reflecting long-term memory. European Biophysics Journal, v. 41, n. 6, p. 505-526, 2012.

ZHAO, Q.; JAYAWARDHANA, D. A.; GUAN, X. Stochastic study of the effect of ionic strength on noncovalent interactions in protein pores. Biophysical Journal, v. 94, n. 4, p. 1267-1275, 2008.

ZHAO, Q. et al. Study of peptide transport through engineered protein channels. The Journal of Physical Chemistry B, v. 113, n. 11, p. 3572-3578, 2009. 